\title{
Sufentanil alleviates cerebral ischemia-reperfusion injury by inhibiting inflammation and protecting the blood-brain barrier in rats
}

\author{
Zhen Wang, ${ }^{1}$ Xiaoyan Du, ${ }^{2}$ Daoyang Yu, ${ }^{1}$ Yang Yang, ${ }^{3}$ Gaoen Ma, ${ }^{4}$ Xueli Jia, ${ }^{1}$ Lulu Cheng \\ ${ }^{1}$ Department of Anesthesiology, Zhumadian Central Hospital, Zhumadian \\ ${ }^{2}$ Rehabilitation Ward, Zhumadian City Welfare Home for Children, Zhumadian \\ ${ }^{3}$ Department of Neurosurgery, Zhumadian Central Hospital, Zhumadian \\ ${ }^{4}$ Department of Ophtalmology, Third Affiliated Hospital of Xinxiang Medical College, Xinxiang \\ ${ }^{5}$ Laboratory of Microneurosurgery, Zhumadian Central Hospital, Zhumadian, China
}

Stroke is a brain system disease with a high fatality rate and disability rate. About $80 \%$ of strokes are ischemic strokes. Cerebral ischemia-reperfusion injury (CIRI) caused by ischemic stroke seriously affects the prognosis of stroke patients. The purpose of this study is to investigate the effect of sufentanil (SUF) on CIRI model rats . We used middle cerebral artery occlusion (MCAO) to make the CIRI model in rats and monitored region cerebral blood flow (rCBF) to ensure that blood flow was blocked and recanalized. We used ELISA and RT-PCR to detect the expression of inflammatory factors in rat serum and brain tissue. In addition, we detected the expression of metalloproteinase (MMP) 2, MMP9 and collagen IV in brain tissues and performed Evans blue (EB) assay to determine the permeability of the blood-brain barrier (BBB). Finally, we clarified the apoptosis of brain tissue through the TUNEL staining and the detection of caspase $3, \mathrm{Bcl} 2$ and Bax. Various concentrations of SUF, especially 5,10 and $25 \mu \mathrm{g} / \mathrm{kg}$ of SUF, all alleviated the infarct size, neurological function and brain edema of MCAO rats. SUF pretreatment also effectively reduced the expression of inflammatory cytokines in MCAO rats, including interleukin (IL)-1 $\beta$, IL-4, IL-6, IL-8, IL-10 and tumor necrosis factor (TNF)- $\alpha$. In addition, SUF also inhibited MMP2 and MMP9 and promoted the expression of collagen IV, indicating that SUF attenuated the destruction of the BBB. SUF also inhibited caspase 3 and Bax rats and promoted $\mathrm{Bcl} 2$ in MCAO rats, thus inhibiting cell apoptosis. SUF pretreatment effectively improved the neurological function and cerebral infarction of $\mathrm{MCAO}$ rats, inhibited excessive inflammation in rats, protected the $\mathrm{BBB}$, and inhibited cell apoptosis in brain tissue.

Key words: Sufentanil; cerebral ischemia-reperfusion injury; inflammation; blood-brain barrier.

Correspondence: Lulu Cheng, MM. Laboratory of Microneurosurgery, Zhumadian Central Hospital, 747 Zhonghua Road, Zhumadian 463000, China. E-mail: 55526649@qq.com

Contributions: All the authors made a substantive intellectual contribution, read and approved the final version of the manuscript and agreed to be accountable for all aspects of the work.

Conflict of interest: The authors declare that they have no competing interests, and all authors confirm accuracy.

Ethics approval and consent to participate: The animal experiment was approved by the Zhumadian Central Hospital Experimental Animal Ethics Committee.

Availability of data and materials: The data used to support the findings of this study are available from the corresponding author on reasonable request. 


\section{Introduction}

Stroke is an acute cerebrovascular disease. ${ }^{1}$ It is a group of diseases that cause sudden cerebral hemorrhage due to rupture of cerebrovascular or ischemia and hypoxia in some areas of the brain due to blockage of cerebrovascular and poor blood flow. ${ }^{1}$ Stroke includes ischemic and hemorrhagic stroke. ${ }^{2}$ The occurrence of stroke is closely related to age, race, hypertension, arteriosclerosis, smoking, drinking, and unhealthy diet. ${ }^{2}$ Brain tissue is highly sensitive to ischemia, and once it occurs, pathological changes will quickly appear, and brain tissue will soon enter a state of irreversible damage. ${ }^{3}$ Tissue recovery and reperfusion therapy not only can not improve the brain tissue ischemia, but cause more serious damage to the brain tissue and its function, that is, cerebral ischemia-reperfusion injury (CIRI). ${ }^{4}$ The prognosis of CIRI patients is usually poor because CIRI of neurons is irreversible. ${ }^{4}$ Therefore, how to prevent and reduce CIRI is still a research hotspot in the medical field.

The concept of anesthetic preconditioning was initially proposed in $1997 .{ }^{5}$ Similar to the phenomenon of ischemic preconditioning, anesthetic treatment given for a certain period of time before ischemia can reduce the organs injury caused by ischemia and has a protective effect on vital organs. ${ }^{6}$ As an opioid analgesic widely used in clinics, sufentanil (SUF) has the strongest analgesic effect, wide safety range, and stable hemodynamics. ${ }^{7}$ SUF, which mainly acts on mu opioid receptors, was originally used as an analgesic. ${ }^{7}$ In recent years, some clinical and animal experimental studies have found that SUF has a good protective effect against ischemia-reperfusion injury. 89 SUF can protect the liver from ischemia-reperfusion injury by its excellent anti-inflammatory effect. ${ }^{8}$ In addition, SUF has also been found to alleviate myocardial ischemia-reperfusion injury by activating the ERK1/2 signaling pathway or miR-125a/DRAM2 axis. ${ }^{10,11}$ A clinical study also shows that SUF can alleviate ischemia-reperfusion injury in patients with coronary heart disease. ${ }^{12}$ Therefore, we speculated that SUF may have a therapeutic effect on ischemia-reperfusion disease. However, there is currently no relevant research on the effect of SUF on CIRI, so the impact of SUF on CIRI is unclear. Therefore, we used SUF to treat CIRI model rats to investigate the potential therapeutic effects of SUF on CIRI.

\section{Materials and Methods}

\section{Animals}

A total of 60 healthy male Sprague Dawley (SD) rats (220-300 g) (Charles River, Beijing, China) were used in this study. The rats were kept in the Specific Pathogen Free (SPF) barrier facility of the Zhumadian Central Hospital Experimental Animal Center. The barrier facility was maintained with the room temperature of $22 \pm 2^{\circ} \mathrm{C}$, the relative humidity of $40-60 \%$ and alternating light for $24 \mathrm{~h}$. Rats can eat and drink freely in cages.

\section{Procedure of middle cerebral artery occlusion (MCAO) model}

After being anesthetized with $2 \%$ sodium pentobarbital (40 $\mathrm{mg} / \mathrm{kg}$ ), the rats were fixed on the operating table. We made a $1 \mathrm{~cm}$ incision in the right of center of the rat's neck. Then we used a tweezer to separate the fascia and muscles of the neck until the common carotid artery is exposed. After ligating the common carotid artery with a thin thread, we inserted the monofilaments from the external carotid artery at the distal end of the ligation into the internal carotid artery. After the monofilaments enter the rat's brain about $25 \mathrm{~mm}$, it can block the middle cerebral artery. After 1 $\mathrm{h}$ of blocking, we quickly removed the monofilaments and loosened the common carotid artery to refill the blood flow in the rat's brain. Finally, we sutured the skin of the rat brain and disinfect the incision with iodophor. ${ }^{13}$ The procedure of the rats in the Sham group was the same as the above, but no ischemia model was established. During the operation, the rat's regional cerebral blood flow (rCBF) was monitored to ensure the success of ischemia and reperfusion. After $1 \mathrm{~h}$ of ischemia and $24 \mathrm{~h}$ of reperfusion, we killed the rats through spinal dislocation and collected the whole brain tissue. One week before surgery, SUF $(5 \mu \mathrm{g} / \mathrm{kg}, 10 \mu \mathrm{g} / \mathrm{kg}$ and $25 \mu \mathrm{g} / \mathrm{kg}$, Sigma-Aldrich, St. Louis, MO, USA) was used to inject subcutaneously into MCAO rats daily. Rats in the Sham group were injected with an equal amount of sterile saline.

\section{Infarct size assessment}

After $1 \mathrm{~h}$ of ischemia and $24 \mathrm{~h}$ of reperfusion, we collected rat brain tissue and placed it in a refrigerator at $-20^{\circ} \mathrm{C}$ for $30 \mathrm{~min}$. Then the frozen brain tissue was cut into $2 \mathrm{~mm}$ thick sections and placed in 2\% 2,3,5-triphenyltetrazolium chloride (TTC) solution (SigmaAldrich, St. Louis, MO, USA). A total of 25 rats were assessed. One brain tissue was cut into 5 pieces for replicates. The TTC solution containing the sections was placed in a $37^{\circ} \mathrm{C}$ constant temperature water bath and incubated for $10 \mathrm{~min}$ in the dark. Then we took out the sections and rinsed them, and then recorded pictures with digital camera. ImageJ 1.80 was used for measurement. Normal brain tissue appears red and infarct tissue appears white. Infarct size $=$ infarct area/total area $\times 100 \%$.

\section{Modified Neurological Severity Score (mNSS) assessment}

After $1 \mathrm{~h}$ of ischemia and $24 \mathrm{~h}$ of reperfusion, we used mNSS to assess nerve damage. A total of 25 rats were assessed. The mNSS score was determined by tests of motor skills, sensory ability, response and balance. The higher the score, the more severe the nerve damage. The mNSS nerve injury classification is as follows: severe injury (score 13-18), moderate injury (score 7-12), mild injury (score 1-6) and no injury (score 0 ). The assessment was carried out by people who did not know the animal group. ${ }^{14}$

\section{Water content examination}

After $1 \mathrm{~h}$ of ischemia and $24 \mathrm{~h}$ of reperfusion, we assessed the degree of cerebral edema in rats by measuring the water content of brain tissue by dry and wet method. A total of 25 rats were used for water content examination. The weight of fresh rat brain tissue is wet weight. The brain tissue was then placed in a $100^{\circ} \mathrm{C}$ incubator for $24 \mathrm{~h}$. The weight of the brain tissue at this time is the dry weight. Brain tissue water content $=($ wet weight-dry weight $) /$ dry weight $\mathrm{x} 100$.

\section{Enzyme-linked immunosorbent assay (ELISA)}

ELISA was used to detect the content of inflammatory factors interleukin (IL)-1b, and tumor necrosis factor (TNF)- $\alpha$ in rat brain tissue and serum. A total of 25 rats were used and there were 3 replicates. The standard in the ELISA kits (Thermo Fisher Scientific, Waltham, MA, USA) was diluted to different concentrations to make a standard curve. Standards and samples were added to the wells of the microtiter plate and incubated at $37^{\circ} \mathrm{C}$ for $2 \mathrm{~h}$. Then we added antibodies to the wells for $1 \mathrm{~h}$ and horseradish peroxidase for $30 \mathrm{~min}$. After washing, the chromogenic solution was added to each well for $20 \mathrm{~min}$ at $37^{\circ} \mathrm{C}$. Finally, we added stop solution and used a microplate reader to detect the absorbance at $450 \mathrm{~nm}$. 


\section{RNA isolation and RT-PCR}

Fifty $\mathrm{mg}$ of brain tissue was collected and dissolved by $1 \mathrm{~mL}$ TRIzol (Invitrogen, Carlsbad, CA, USA). The chloroform-isopropanol method was used to extract total RNA. We used reverse transcription kits (Vazyme, Nanjing, Jiangsu, China) to reverse mRNA to complementary deoxyribose nucleic acid (cDNA). The reverse transcription system included $10 \mu \mathrm{L}$ of $5 \times$ Reaction Buffer, $2.5 \mu \mathrm{L}$ of dNTP, $2.5 \mu \mathrm{L}$ of RTase, $1.25 \mu \mathrm{L}$ of RRI and $3.75 \mu \mathrm{L}$ of Rnase Free water. The cDNA was stored in a refrigerator at $-20^{\circ} \mathrm{C}$. SYBR Green Master Mix (Vazyme, Nanjing, Jiangsu, China) was used for PCR. The PCR system included $0.4 \mu \mathrm{L}$ of forward primer, $0.4 \mu \mathrm{L}$ of reverse primer, $10 \mu \mathrm{L}$ of Mix, $2 \mu \mathrm{L}$ of cDNA and $7.2 \mu \mathrm{L}$ of Rnase Free water. glyceraldheyde 3-phosphate dehydrogenase (GAPDH) was used as an internal control. $2^{-\Delta \Delta C T}$ was used to indicate the relative expression of mRNA. The primer sequences were shown in Table 1. A total of 25 rats were used in this experiment and there were 3 replicates.

\section{Evans blue (EB) assay}

Five $\mathrm{h}$ before the rats were sacrificed, we injected $0.1 \mathrm{~mL}$ of $2 \%$ EB (Sigma-Aldrich) into rats through the tail vein, followed by infusion with normal saline. After killing the rats, we collected brain tissue and weighed it. We added a corresponding volume of phosphate buffered saline (PBS) according to the weight of the collected brain tissue to prepare homogenate $(0.1 \mathrm{~g} / \mathrm{mL})$ by centrifugation $(15,000 \mathrm{rpm}, 30 \mathrm{~min})$. Then we collected $0.5 \mathrm{~mL}$ of supernatant and add an equal volume of trichloroacetic acid, and incubated them overnight at $4{ }^{\circ} \mathrm{C}$. The next day we took out the mixture and placed it in a centrifuge for centrifugation $(15,000 \mathrm{rpm}, 30$ $\min$ ). Then we collected the supernatant and used a microplate reader to measure the absorbance at $615 \mathrm{~nm}$. A total of 25 rats were used in this experiment and there were 3 replicates.

\section{TdT-mediated dUTP Nick-End Labeling (TUNEL) staining}

Rat brain tissues were collected and fixed for $24 \mathrm{~h}$ with $4 \%$ paraformaldehyde. The brain tissue was then made into paraffin blocks and cut into paraffin sections with a thickness of $5 \mu \mathrm{m}$. The TUNEL apoptosis kit (Sigma-Aldrich) was used to detect paraffin sections. After deparaffinization and hydration, the paraffin sections were placed in a wet box and a mixture of TdT and DIG-DUTP was dropped on the sections. The wet box was placed in a refrigerator at $4^{\circ} \mathrm{C}$. After $2 \mathrm{~h}$, the blocking solution was dropped on the tissue for blocking at room temperature for $30 \mathrm{~min}$. The antibody dilution was then dropped on the sections for $40 \mathrm{~min}$. Finally, we washed the sections and mounted them with anti-fluorescence quenching mounting medium. The level of apoptosis was assessed by calculating the percentage of positive cells. One section per animal was used and four microscope fields per section were used to calculate the average value. A total of 15 rats were used in this experiment and there were 3 replicates.

\section{Statistical analysis}

Statistical Product and Service Solutions (SPSS) 20.0 (IBM, Armonk, NY, USA) was used to analyze the data of this study and Graphpad Prism 7.0 (La Jolla, CA, USA) was used to make graphs. The measurement data were represented by the mean \pm standard deviation. Comparison of data between multiple groups was performed using one-way analysis of variance followed by post-hoc test (Bonferroni); $\mathrm{p}<0.05$ was considered as statistically significant.

\section{Results}

\section{SUF alleviated brain damage in MCAO rats during ischemia and reperfusion}

In order to clarify the effect of SUF on the brain tissue function of rats after CIRI, we used MCAO method to establish a CIRI model of rats, and test the condition of cerebral infarction and nerve function of the rats in the ischemic phase ( $1 \mathrm{~h}$ after ischemia) and reperfusion phase ( $24 \mathrm{~h}$ after reperfusion). In order to determine the optimal concentration of SUF, we used different concentrations of SUF $(1,5,10,25,50 \mu \mathrm{g} / \mathrm{kg})$ to treat MCAO rats. We first determined the occlusion and recanalization of cerebral arteries by monitoring the condition of rat $\mathrm{rCBF}$. As shown in Figure $1 \mathrm{~A}$, the rCBF of rats in the Sham group remained at a normal level throughout. In MCAO rats, the rCBF dropped sharply to about 20 pu after blocking the cerebral artery and returned to normal after recanalization. The results of $\mathrm{rCBF}$ indicated that the MCAO model was successfully established. The results of TTC staining showed that MCAO rats had obvious cerebral infarction, especially in the reperfusion period. SUF was found to ameliorate cerebral infarction in MCAO rats during the ischemic and reperfusion phases (Figure 1B). The results of mNSS indicated that SUF can improve the neurological function of MCAO rats during the ischemic phase and reperfusion phase (Figure 1C). In addition, the brain water content of MCAO rats increased significantly, and SUF pretreatment can reduce the water content and relieve brain edema (Figure 1D). Among the different concentrations of SUF, the 5,10 and $25 \mu \mathrm{g} / \mathrm{kg}$ of SUF had better results.

\section{SUF reduced inflammation levels in MCAO rats}

Excessive inflammation is one of the important causes of CIRI, so we detected changes in the level of inflammation in MCAO rats. The above results indicated that the 5, 10 and 25 $\mu \mathrm{g} / \mathrm{kg}$ of SUF had good results, so we used these three concentrations of SUF to treat rats in the subsequent experiment. We collected the brain tissue and serum of rats and detected the concentration of IL-1 $\beta$ (Figure 2A) and TNF- $\alpha$ (Figure 2B) by ELISA. ELISA results showed that SUF effectively reduced the concentration of IL- $1 \beta$ and TNF- $\alpha$ in brain tissue and serum. In addition, we isolated total RNA in rat brain tissue and detected the changes in mRNA of various inflammatory factors. The mRNAs of IL-1 $\beta$, IL-4, IL-6,

Table 1. Primer sequences.

\begin{tabular}{lcc} 
Names & \multicolumn{1}{c}{ Forward } & \multicolumn{1}{c}{ Reverse } \\
GAPDH & ATGGCTACAGCAACAGGGT & TTATGGGGTCTGGGATGG \\
$I L-1 \beta$ & CCCTTGACTTGGGCTGT & CGAGATGCTGCTGTGAGA \\
\hline$I L-4$ & GGCAACAAGGAACACCAC & CACCGAGACCCCAGAC \\
$I L-6$ & CACCAGGAACGAAAGCAA & CAACAACATCAGTCCCAAGA \\
\hline$I L-8$ & GAGCAACCCATACCCATCGA & TGGTCCCACCATATCTTCTTAATCT \\
$I L-10$ & AGGGTTACTTGGGTTGCC & GGGTCTTCAGCTTCTCTCC \\
\hline TNF- $\alpha$ & CAGCCAGGAGGGAGAC & GTATGAGAGGGACGGAACC \\
MMP2 & TTGACCAGAACACCATCG & CTTGCGGGGAAAAAGT \\
\hline MMP9 & CGCTGGGCTTAGATCATT & TGCTGGATGCCTTTATGT \\
Collagen IV & GCCACCATAGAGAGAAGCG & CAACAGGAGGGGAGAGGA \\
\hline Caspase 3 & GGAACGCGAAGAAAATG & ATTTTGAATCCACGGAGGT \\
Bcl2 & GAGGTCTTCTTCCGTGTGG & GATCAGCTCGGGCACTTT \\
\hline Bax & AGGAACTCTTCAGGGATGG & GCGATGTTGTCCACCAG \\
\hline
\end{tabular}


IL-8, IL-10 and TNF- $\alpha$ in MCAO rats all increased significantly. The three concentrations of SUF all reduced the expression of proinflammatory factors (IL- $1 \beta$, IL-6, IL-8, and TNF- $\alpha$ ) mRNA in rat brain tissue to varying degrees (Figure $2 \mathrm{C}-2 \mathrm{H}$ ). These results indicated that SUF pretreatment can significantly improve the level of inflammation in MCAO rats.

\section{SUF alleviated the damage of blood-brain barrier in MCAO rats}

CIRI is accompanied by an increase in the permeability of the $\mathrm{BBB}$, which further leads to aggravation of CIRI. Therefore, protecting the $\mathrm{BBB}$ is essential to alleviate CIRI. We detected the mRNA expression of metalloproteinase (MMP) 2 (Figure 3A), MMP9 (Figure 3B) and collagen IV (Figure 3C) in the brain tissue of rats and found that the mRNA of MMP2 and MMP9 increased significantly and collagen IV decreased in the brain tissue of MCAO rats, which implied that the increase in permeability of BBB of MCAO rats. The mRNA levels of MMP2 and MMP9 in the brain tissue of MCAO rats pretreated with SUF were lower than those of untreated rats and mRNA of collagen IV increased in rats treated with SUF, which indicated that SUF pretreatment protected the $\mathrm{BBB}$ of rats. In addition, we also found through $\mathrm{EB}$ assay that SUF pretreatment reduced the permeability of the BBB of MCAO rats, which was manifested as a decrease in EB (Figure $3 \mathrm{D})$.

\section{SUF inhibited cell apoptosis in the brain tissue of MCAO rats}

CIRI can cause a large number of cell apoptosis and necrosis in brain tissue, which lead to irreversible damage. Therefore, we detected the changes of cell apoptosis in rat brain tissue. TUNEL detected cell apoptosis in the ischemic penumbra of rat brain tissue. There were a large number of positive spots in the brain tissue sections of MCAO rats, while the positive spots in the tissue sections of SUF pretreated rats were significantly reduced (Figure 4 A,B). This indicated that SUF pretreatment reduced the apoptosis rate of the brain tissue of MCAO rats. The mRNA levels of caspase 3 (Figure 4C), Bcl2 (Figure 4D) and Bax (Figure 4E) in brain tissues
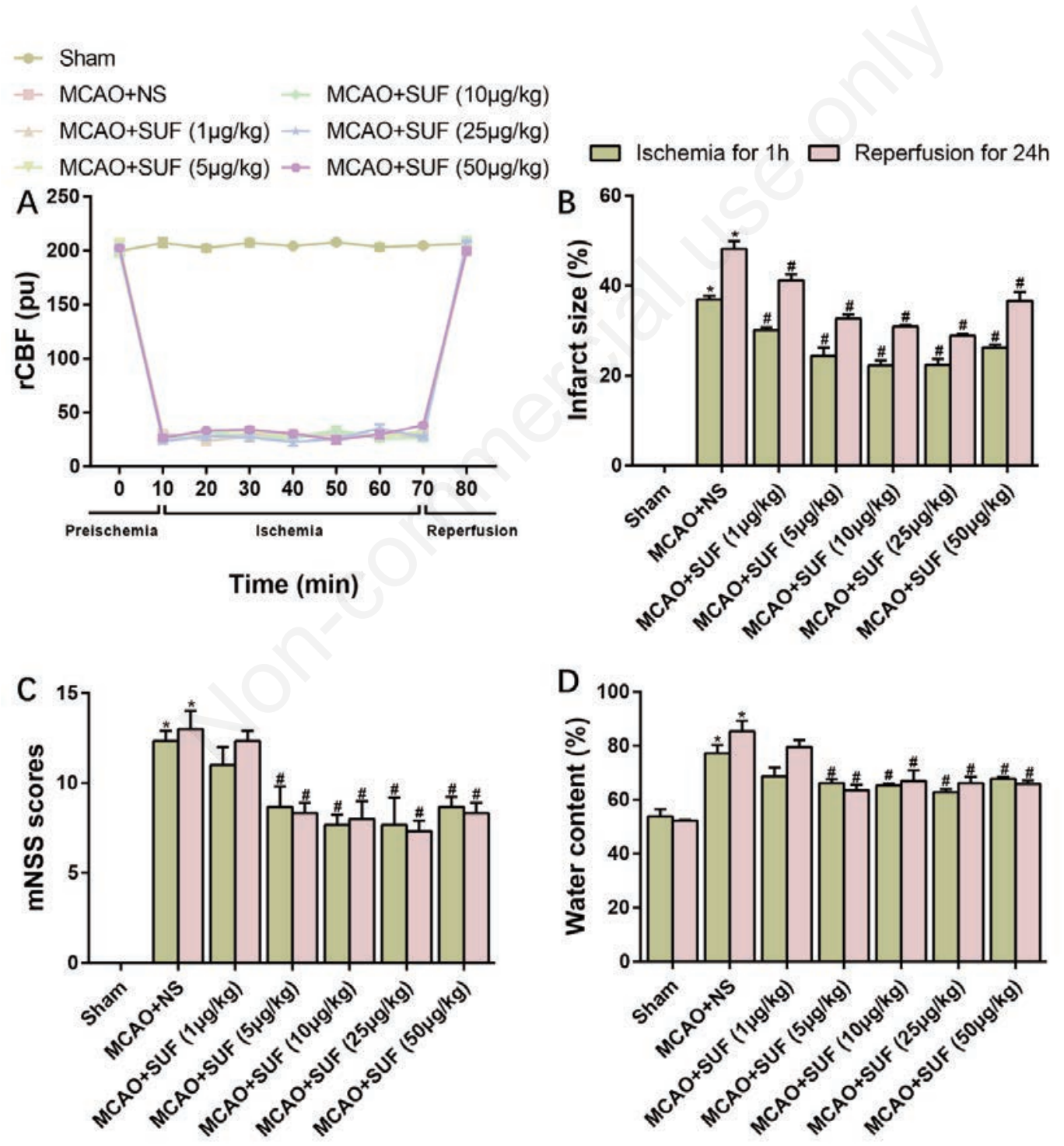

Figure 1. SUF alleviated brain damage in MCAO rats during ischemia and reperfusion. A) Regional cerebral blood flow (rCBF) was monitored during modeling. B) Infarct size of brain tissue in rats. C) Modified neurological severity score ( $\mathrm{mNSS}$ ) of rats in seven groups. D) Cerebral water content of rats in seven groups. ${ }^{*} \mathrm{p}<0.05$ vs Sham group; \#p<0.05 vs MCAO+NS group. 

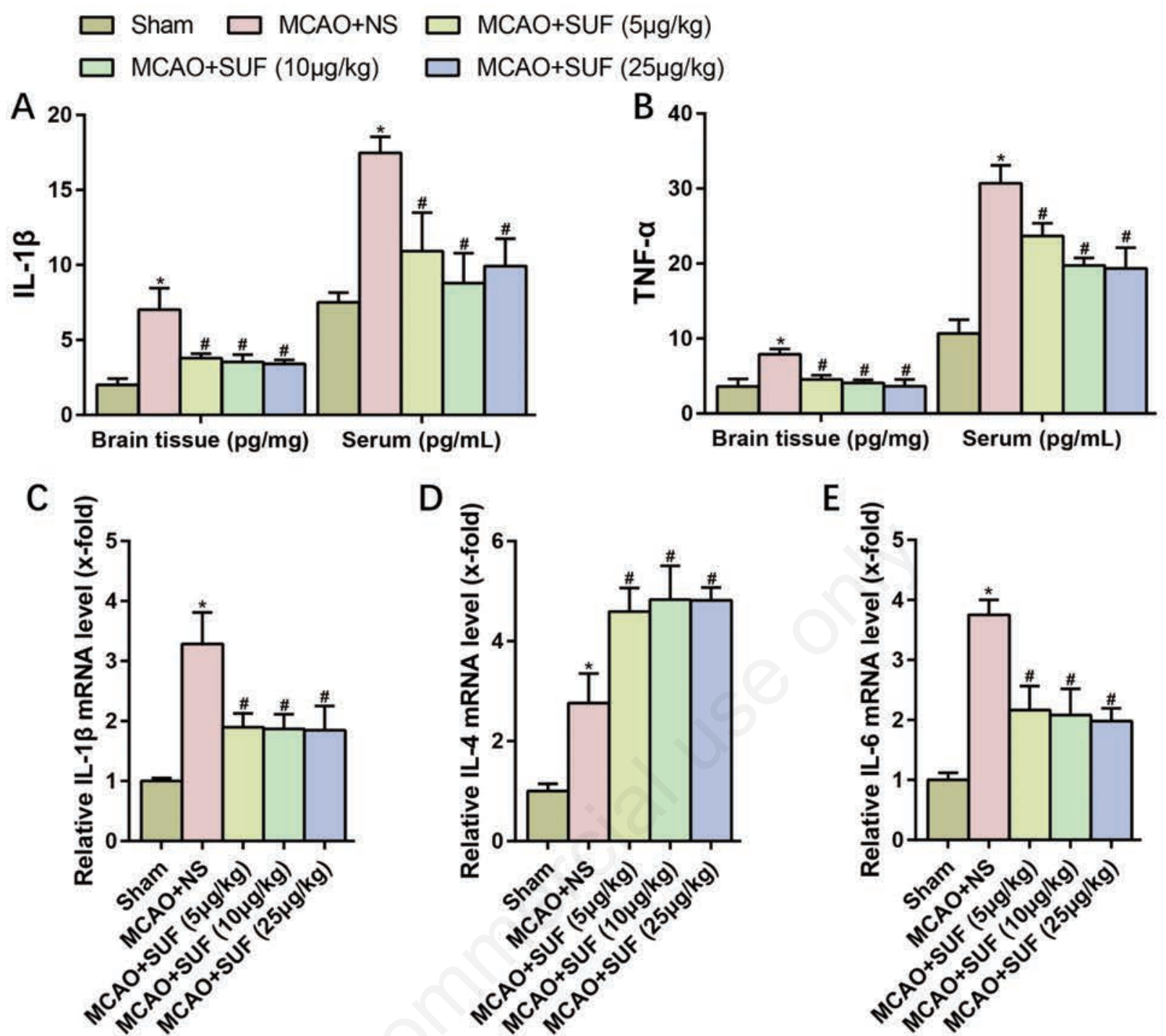

E
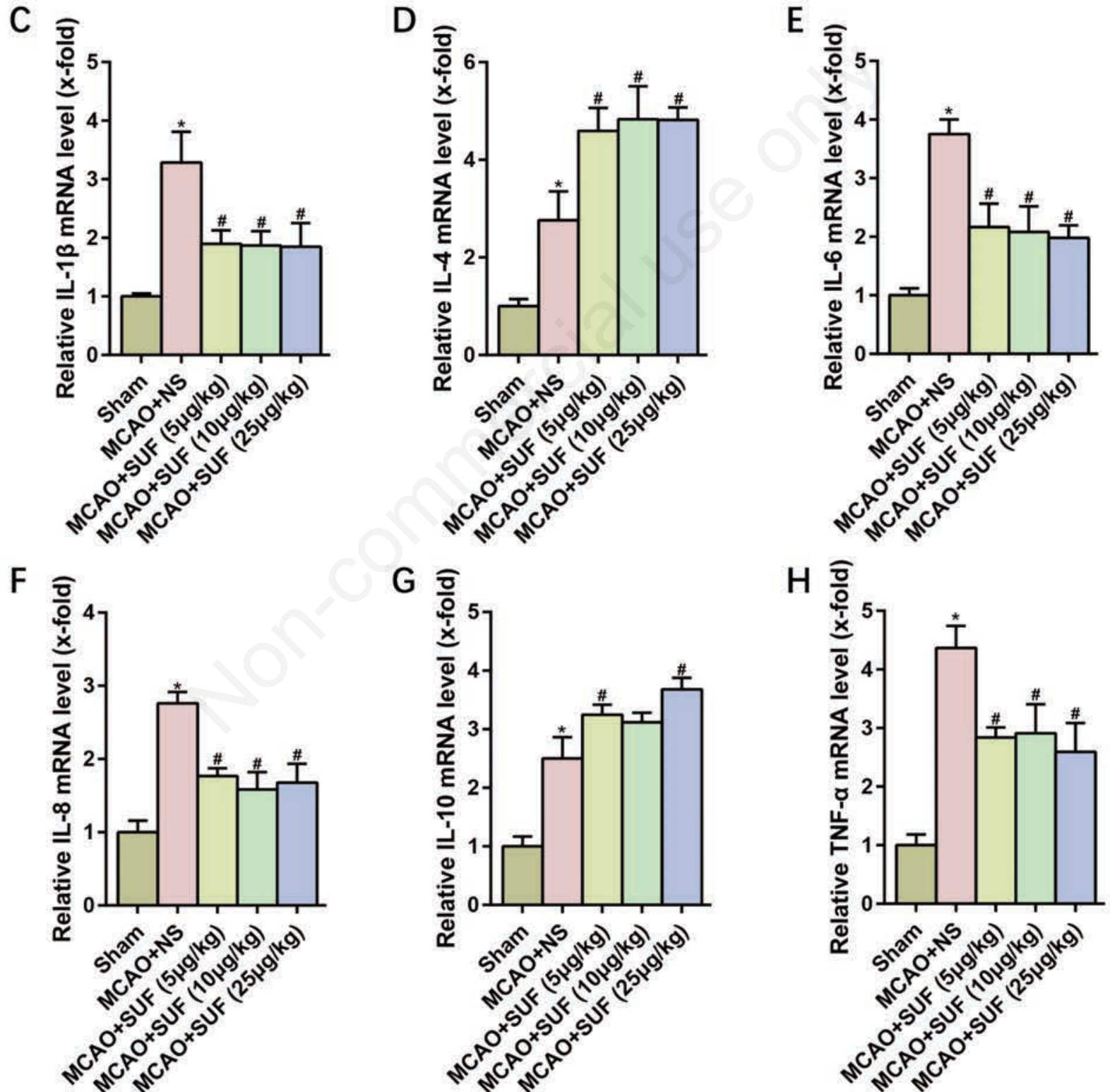

Figure 2. SUF reduced inflammation levels in MCAO rats. A,B) The concentrate of IL-1 $\beta$ and TNF- $\alpha$ in brain tissue and serum of rats in four groups. C-H) The mRNA expression of IL-1 $\beta$, IL-4, IL-6, IL-8, IL-10 and TNF- $\alpha$ in brain tissue. ${ }^{*} p<0.05 v s$ Sham group; $\# \mathbf{p}<\mathbf{0 . 0 5}$ vs MCAO+NS group. 
were also detected and it was found that SUF pretreatment can inhibit caspase 3 and Bax and promote the expression of $\mathrm{Bcl} 2$ mRNA. These results indicated that SUF pretreatment inhibited the cell apoptosis in rat brain tissue after CIRI.

\section{Discussion}

In recent years, with the gradual aging of the population, ischemic cerebrovascular diseases have become one of the main factors threatening the health of elderly patients, and it has become a research hot topic in modern medical practice. ${ }^{15}$ Cerebral ischemic diseases induce irreversible damage to the brain. Long- term reperfusion injury will cause the expansion of the cerebral infarct size, and clinically the method of recovering and recanalizing brain blood cannot fundamentally solve the above problems. ${ }^{16}$ Drug pretreatment is a hot spot in today's research. ${ }^{17}$ The protective effects of anesthetics pretreatment are obvious, including opioids, inhalation anesthetics and propofol. These anesthetics have been proven in animal experiments. ${ }^{18,19}$ Morphine is a representative of opioids, and the research on its pretreatment is relatively mature, especially on its protective effect on the heart. ${ }^{18}$ The protective effect of opioid pretreatment is mainly mediated by opioid receptors. ${ }^{19}$ The most commonly used opioids in clinical are receptor agonists, including fentanyl, SUF, remifentanil and alfentanil, all of which have the effect of drug pretreatment. ${ }^{20}$ Compared with
A

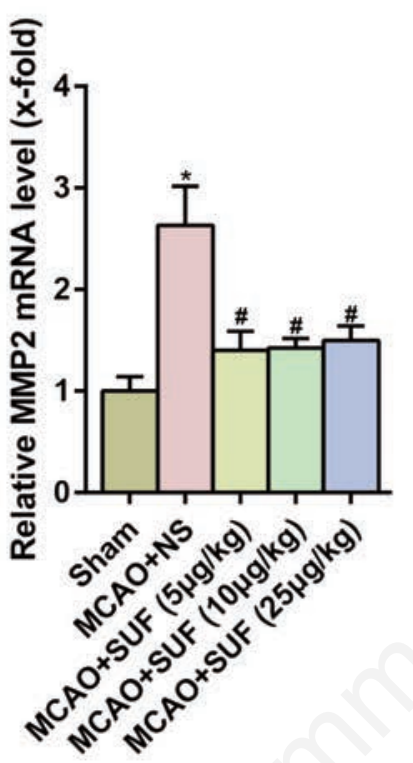

C

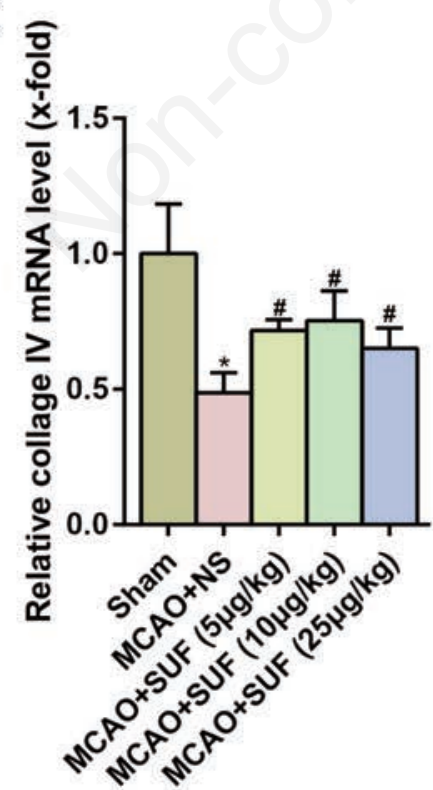

B

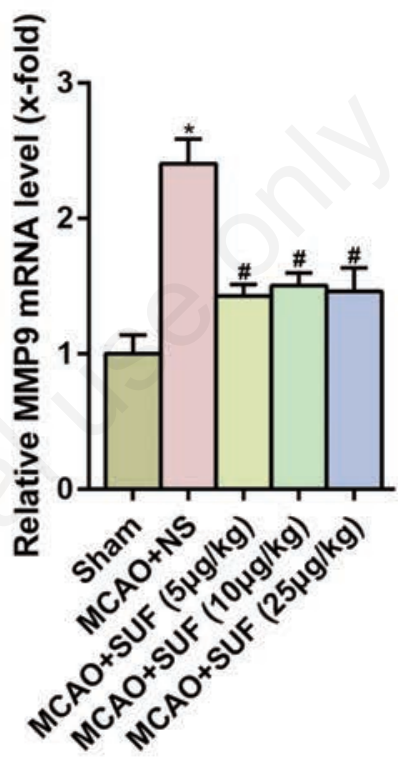

D

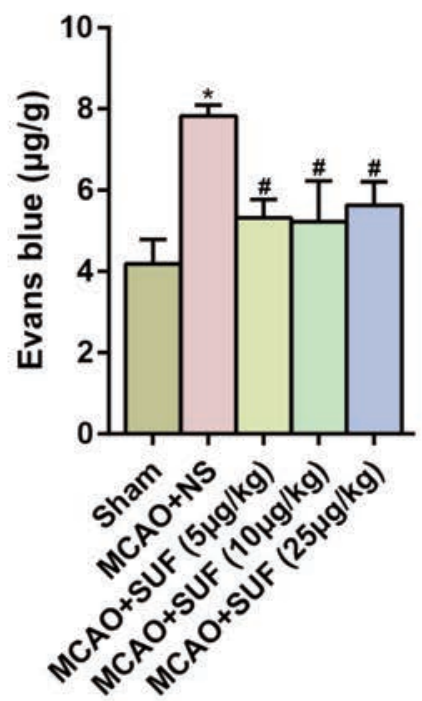

Figure 3. SUF alleviated the damage of blood-brain barrier in MCAO rats. A-C) The mRNA expression of MMP2, MMP9 and collagen IV in rats. D) Evans blue in rats of four groups. ${ }^{*} \mathrm{p}<0.05$ vs Sham group; $\# \mathrm{p}<0.05$ vs $\mathrm{MCAO}+\mathrm{NS}$ group. 
fentanyl, SUF has the advantages of stronger lipophilicity, higher plasma protein binding rate, relatively smaller volume of distribution, strong opioid receptor affinity and longer duration of action. ${ }^{21}$ But so far, there are few reports about the protective effect of opioid pretreatment on the brain. In this experimental study, a rat CIRI model was established, and various doses of SUF were used at the same time. The results showed that SUF at 5,10 and $25 \mu \mathrm{g} / \mathrm{kg}$ can effectively improve the neurological function of MCAO rats and reduce the level of inflammation and the permeability of the BBB in rats. This indicated that SUF may have a potential therapeutic effect on CIRI.

Inflammatory mediators in the brain are upregulated after cerebral ischemia. ${ }^{22}$ They are not only expressed in cells of the immune system, but also in brain cells including glial cells and neurons. ${ }^{22}$
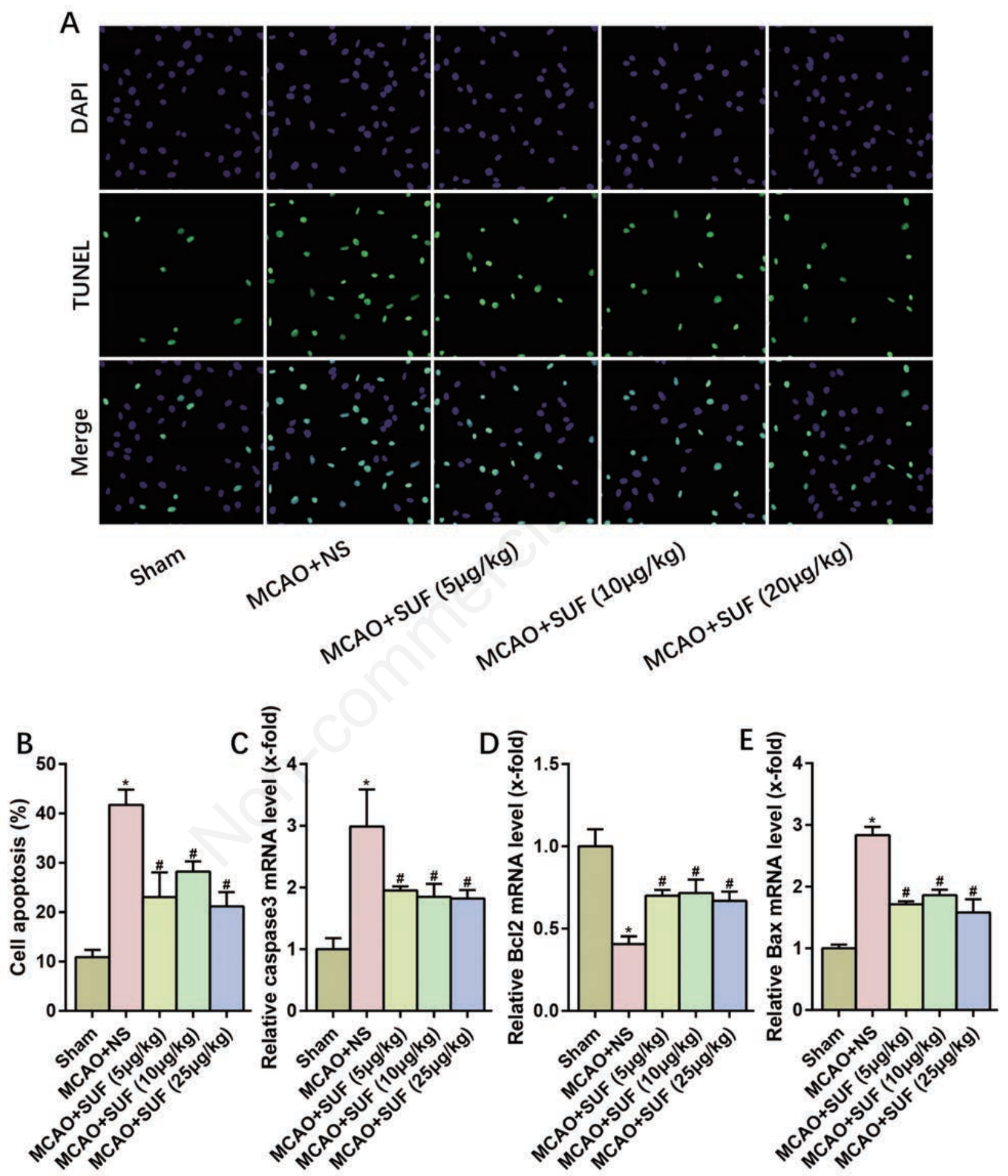

Figure 4. SUF inhibited cell apoptosis in the brain tissue of MCAO rats. A,B) TUNEL staining results of brain tissue in rats (magnification: 200x) and quantification. C-E) The mRNA expression of caspase 3, Bcl-2 and Bax in brain tissue. ${ }^{*} \mathrm{p}<0.05$ vs Sham group; \#p $<0.05$ vs MCAO+NS group. 
The most important cytokines associated with cerebral ischemia inflammation are IL-1 $\beta$, TNF- $\alpha$, IL-6, IL-10 and transforming growth factor- $\beta$ (TGF- $\beta$ ). Among these cytokines, IL- $1 \beta$ and TNF$\alpha$ are closely related to brain injury. ${ }^{23}$ The early stage of inflammatory injury after cerebral ischemia-reperfusion is mainly caused by the pro-inflammatory mediator TNF- $\alpha .{ }^{24}$ This cytokine is synthesized by macrophages, especially activated microglia. TNF- $\alpha$ activates neutrophils and lymphocytes and promotes the synthesis of adhesion molecules and other inflammatory mediators such as IL$1 \beta$ and IL-6. ${ }^{24}$ Parker et al. ${ }^{25}$ found that compared with wild-type mice, IL-1 $\beta$-deficient mice have a smaller infarct size. In addition, treatment with IL-1 receptor antagonists can reduce infarct size. The level of TNF- $\alpha$ in the brain is also upregulated after ischemia, and its expression is similar to IL- $1 \beta$. Inhibition of TNF- $\alpha$ can reduce ischemic brain injury, and administration of recombinant TNF- $\alpha$ protein after stroke can aggravate ischemic brain injury. ${ }^{26}$ SUF has been found to reduce the level of inflammation in a variety of injury models. Hu et al. revealed that SUF can reduce IL-1b, IL-6 and TNF-a in rats with acute lung injury. ${ }^{27}$ Lian et al. also found that it inhibits inflammatory damage during liver ischemiareperfusion. ${ }^{8,9}$ Our results also found that the expression of a large number of inflammatory cytokines in the MCAO model rats was significantly increased, which indicated that the inflammatory response was involved in the brain injury of the MCAO model rats. The inflammatory cytokines of SUF pretreated rats were significantly downregulated, indicating that SUF effectively alleviated the brain injury of MCAO rats by inhibiting inflammation. In addition, SUF also promoted the upregulation of anti-inflammatory factors IL-4 and IL-10, which further inhibited the inflammatory response in MCAO rats. These results are consistent with the results of other previous studies. ${ }^{24-26}$

Cerebral edema mainly includes cytotoxic brain edema, vasogenic brain edema, interstitial brain edema and mixed brain edema. ${ }^{28}$ Vascular brain edema is related to the increase of BBB permeability. ${ }^{29}$ The early stage of cerebral ischemia-reperfusion is accompanied by the expression of a variety of inflammatory cytokines and adhesion molecules, which promotes the transformation of cerebral ischemic injury to inflammatory injury. ${ }^{30}$ The aggregation and infiltration of polymorphonuclear leukocytes produce a large number of proteolytic enzymes, oxygen free radicals and other effector molecules, which cause damage to the capillary endothelial cells and basement membrane of brain tissue, and induce vascular brain edema and hemorrhage. ${ }^{31}$ MMPs mainly include members such as MMP2 and MMP9. MMPs are a family of metalloproteinase endonucleases that are zinc ion-dependent. They can degrade most of the extracellular matrix components. ${ }^{31}$ MMPs, especially MMP9, can cause the destruction of the tight junctions between brain tissue capillaries and BBB endothelial cells in ischemia-reperfusion, especially BBB microvascular basement membrane, which can cause vasogenic brain edema secondary to cerebral ischemia-reperfusion. ${ }^{31}$ Collagen IV is one of the main components of the basement membrane of the BBB. Collagen IV degrades rapidly after CIRI, which is consistent with the trend of continuous increase in BBB permeability. ${ }^{32}$ Collagen IV, MMPs, BBB and neurovascular unit are closely related. Research results in recent years have shown that inhibiting and knocking out the MMP9 gene have a protective effect on experimental cerebral ischemia and reperfusion. ${ }^{33}$ Rosell et al. ${ }^{33}$ studied the relationship between MMP2/9 in the cerebral ischemic area and the destruction of the BBB after hemorrhagic transformation of ischemic stroke, and the results showed that the level of MMP9 in hemorrhagic and non-hemorrhagic infarct tissues was higher than that in the contralateral side; the expression level of MMP9 in the area was significantly higher than that in the non-bleeding area and the contralateral area; the level of MMP2 did not change; the infiltration of surrounding cerebral capillaries by MMP9-positive neutrophils was related to serious degradation of basement membrane collagen IV and blood extravasation. The study showed that there is a large number of neutrophil infiltrations in the infarct and hemorrhage area, and the local MMP9 level is higher, which is closely related to the degradation of the basement membrane collagen IV and the destruction of the $\mathrm{BBB} .{ }^{33}$ Therefore, protecting the $\mathrm{BBB}$ is one of the keys to the treatment of CIRI. We studied the effects of SUF on MMP2, MMP9 and collagen IV in MCAO rats. SUF was found to inhibit MMP2 and MMP9 and promote the secretion of collagen IV. These results showed that SUF can protect the $\mathrm{BBB}$, and the results of the $\mathrm{EB}$ experiment also show that SUF can attenuate the destruction of the BBB by CIRI.

Apoptosis is different from necrosis. Apoptosis caused by reperfusion injury is mainly distributed in the peripheral area of cerebral infarction. ${ }^{34}$ Apoptosis can also exacerbate reperfusion injury. ${ }^{34} \mathrm{Bcl} 2 / \mathrm{Bax}$ determines the viability of cells after apoptosis stimulation. A high proportion of $\mathrm{Bcl} 2 / \mathrm{Bax}$ can lead to better antiapoptotic ability of cells. The activation of caspase 3 can reduce DNA damage repair enzymes and increase endonucleases and cell apoptosis. ${ }^{34}$ Some studies have revealed that SUF can reduce the apoptotic level of mesenchymal stem cells and hepatocytes. ${ }^{8,353}$ Our results also show that SUF has a significant inhibitory effect on neuronal apoptosis induced by CIRI. The expression of caspase 3 in the brain tissue of SUF-treated rats decreased significantly and $\mathrm{Bcl} 2 / \mathrm{Bax}$ increased. This indicates that SUF improves the apoptosis level in the brain tissue of MCAO rats.

To sum up, SUF is first to be used in rat CIRI models and this is also the first study to investigate the effect of SUF pretreatment on CIRI in MCAO rats. SUF pretreatment for one week effectively alleviated the neurological damage, cerebral infarction and cerebral edema in MCAO rats. In addition, SUF also inhibited the excessive inflammation caused by CIRI and weakened the destruction of the BBB, and inhibited the apoptosis of brain tissue. SUF may become a potential therapeutic drug for CIRI. We hope that the results of this study can contribute to the clinical treatment of CIRI.

\section{References}

1. Elkind M, Boehme AK, Smith CJ, Meisel A, Buckwalter MS. Infection as a stroke risk factor and determinant of outcome after stroke. Stroke 2020;51:3156-68.

2. Boot E, Ekker MS, Putaala J, Kittner S, De Leeuw FE, Tuladhar AM. Ischaemic stroke in young adults: a global perspective. J Neurol Neurosurg Psychiatry 2020;91:411-7.

3. Oie LR, Kurth T, Gulati S, Dodick DW. Migraine and risk of stroke. J Neurol Neurosurg Psychiatry 2020;91:593-604.

4. Bavarsad K, Barreto GE, Hadjzadeh MA, Sahebkar A. Protective effects of curcumin against ischemia-reperfusion injury in the nervous system. Mol Neurobiol 2019;56:1391404.

5. Cason BA, Gamperl AK, Slocum RE, Hickey RF. Anestheticinduced preconditioning: previous administration of isoflurane decreases myocardial infarct size in rabbits. Anesthesiology 1997;87:1182-90.

6. Nunes RR, Duval NG, de Alencar JC, Franco SB, de Andrade NQ, Dumaresq DM, et al. Anesthetics, cerebral protection and preconditioning. Braz J Anesthesiol 2013;63:119-28.

7. Frey UH, Klaassen M, Ochsenfarth C, Murke F, Thielmann M, Kottenberg E, et al. Remote ischaemic preconditioning increases serum extracellular vesicle concentrations with altered micro-RNA signature in CABG patients. Acta Anaesthesiol Scand 2019;63:483-92.

8. Lian YH, Fang J, Zhou HD, Jiang HF, Xie KJ. Sufentanil pre- 
conditioning protects against hepatic ischemia-reperfusion injury by suppressing inflammation. Med Sci Monit 2019;25:2265-73.

9. Zhou L, Yang X, Shu S, Wang S, Guo F, Yin Y, et al. Sufentanil protects the liver from ischemia/reperfusion-induced inflammation and apoptosis by inhibiting ATF4-induced TP53BP2 expression. Inflammation 2021;44:1160-1174.

10. Tao H, Nuo M, Min S. Sufentanil protects the rat myocardium against ischemia-reperfusion injury via activation of the ERK1/2 pathway. Cytotechnology 2018;70:169-76.

11. Wu Q, Shang Y, Bai Y, Wu Y, Wang H, Shen T. Sufentanil preconditioning protects against myocardial ischemia/reperfusion injury via miR-125a/DRAM2 axis. Cell Cycle 2021;20:383-391.

12. Khosravi MB, Kahrom M, Tahari M, Alizadeh K, Soltani G, Ghanad MA. Effect of the aortic root infusion of sufentanil on ischemia-reperfusion injury in patients undergoing coronary artery bypass grafting: A randomized clinical trial. J Tehran Heart Cent 2019;14:177-182.

13. Li Z, Yulei J, Yaqing J, Jinmin Z, Xinyong L, Jing G, et al. Protective effects of tetramethylpyrazine analogue Z-11 on cerebral ischemia reperfusion injury. Eur $\mathrm{J}$ Pharmacol 2019;844:156-64.

14. Akpınar H, Nazıroğlu M, Övey İS, Çiğ B, Akpınar O. The neuroprotective action of dexmedetomidine on apoptosis, calcium entry and oxidative stress in cerebral ischemia-induced rats: Contribution of TRPM2 and TRPV1 channels. Sci Rep 2016;6:37196.

15. Magalhaes JE, Sampaio RP. Migraine and cerebrovascular diseases: Epidemiology, pathophysiological, and clinical considerations. Headache 2018;58:1277-86.

16. Nguyen H, Aum D, Mashkouri S, Rao G, Vega GJ, Reyes S, et al. Growth factor therapy sequesters inflammation in affording neuroprotection in cerebrovascular diseases. Expert Rev Neurother 2016;16:915-26.

17. Sharma D, Maslov LN, Singh N, Jaggi AS. Remote ischemic preconditioning-induced neuroprotection in cerebral ischemiareperfusion injury: Preclinical evidence and mechanisms. Eur J Pharmacol 2020;883:173380.

18. He SF, Jin SY, Wu H, Wang B, Wu YX, Zhang SJ, et al. Morphine preconditioning confers cardioprotection in doxorubicin-induced failing rat hearts via ERK/GSK-3beta pathway independent of PI3K/Akt. Toxicol Appl Pharmacol 2015;288:349-58.

19. Fassini A, Scopinho AA, Fortaleza E, Resstel L, Correa F. Kappa-opioid receptors in the medial amygdaloid nucleus modulate autonomic and neuroendocrine responses to acute stress. Eur Neuropsychopharmacol 2021;43:25-37.

20. Wojciechowski P, Andrzejewski K, Kaczynska K. Intracerebroventricular neuropeptide FF diminishes the number of apneas and cardiovascular effects produced by opioid receptors' activation. Int J Mol Sci 2020;21:8931.

21. Reardon CE, Kane-Gill SL, Smithburger PL, Dasta JF. Sufentanil sublingual tablet: A new option for acute pain management. Ann Pharmacother 2019;53:1220-6.

22. Stegner D, Klaus V, Nieswandt B. Platelets as modulators of cerebral ischemia/reperfusion injury. Front Immunol 2019;10:2505.

23. Enzmann G, Kargaran S, Engelhardt B. Ischemia-reperfusion injury in stroke: impact of the brain barriers and brain immune privilege on neutrophil function. Ther Adv Neurol Disord 2018;11:1756286418794184.

24. Tuttolomondo A, Di Sciacca R, Di Raimondo D, Renda C, Pinto A, Licata G. Inflammation as a therapeutic target in acute ischemic stroke treatment. Curr Top Med Chem 2009;9:124060.

25. Parker LC, Luheshi GN, Rothwell NJ, Pinteaux E. IL-1 beta signalling in glial cells in wildtype and IL-1RI deficient mice. Br J Pharmacol 2002;136:312-20.

26. Kim E, Kim HC, Lee S, Ryu HG, Park YH, Kim JH, et al. Dexmedetomidine confers neuroprotection against transient global cerebral ischemia/reperfusion injury in rats by inhibiting inflammation through inactivation of the TLR-4/NFkappaB pathway. Neurosci Lett 2017;649:20-7.

27. Hu Q, Wang Q, Han C, Yang Y. Sufentanil attenuates inflammation and oxidative stress in sepsis-induced acute lung injury by downregulating KNG1 expression. Mol Med Rep 2020;22:4298-306.

28. Bai J, Lyden PD. Revisiting cerebral postischemic reperfusion injury: new insights in understanding reperfusion failure, hemorrhage, and edema. Int J Stroke 2015;10:143-52.

29. Jia SW, Liu XY, Wang SC, Wang YF. Vasopressin hypersecretion-associated brain edema formation in ischemic stroke: Underlying mechanisms. J Stroke Cerebrovasc Dis 2016;25:1289-300.

30. Xiao F. Bench to bedside: brain edema and cerebral resuscitation: the present and future. Acad Emerg Med 2002;9:933-46.

31. Rosenberg GA, Yang Y. Vasogenic edema due to tight junction disruption by matrix metalloproteinases in cerebral ischemia. Neurosurg Focus 2007;22:E4.

32. Anik I, Kokturk S, Genc H, Cabuk B, Koc K, Yavuz S, et al. Immunohistochemical analysis of TIMP-2 and collagen types I and IV in experimental spinal cord ischemia-reperfusion injury in rats. J Spinal Cord Med 2011;34:257-64.

33. Rosell A, Cuadrado E, Ortega-Aznar A, Hernandez-Guillamon M, Lo EH, Montaner J. MMP-9-positive neutrophil infiltration is associated to blood-brain barrier breakdown and basal lamina type IV collagen degradation during hemorrhagic transformation after human ischemic stroke. Stroke 2008;39:1121-6.

34. Hausburg MA, Banton KL, Roman PE, Salgado F, Baek P, Waxman MJ, et al. Effects of propofol on ischemia-reperfusion and traumatic brain injury. J Crit Care 2020;56:281-87.

35. Li L, Sun Y, Zhang N, Qiu X, Wang L, Luo Q. By regulating miR-182-5p/BCL10/CYCS, sufentanil reduces the apoptosis of umbilical cord mesenchymal stem cells caused by ropivacaine. Biosci Trends 2019;13:49-57.

Received for publication: 10 September 2021. Accepted for publication: 19 December 2021.

This work is licensed under a Creative Commons Attribution-NonCommercial 4.0 International License (CC BY-NC 4.0).

(C) Copyright: the Author(s), 2022

Licensee PAGEPress, Italy

European Journal of Histochemistry 2022; 66:3328

doi:10.4081/ejh.2022.3328 